\title{
A Methodical Study for Time-Frequency Analysis Model with Experimental Case Study on Chirp Signal
}

\author{
Qasem Abu Al-Haija* \\ Electrical and Computer Engineering Department, Tennessee State University, Nashville/TN, USA
}

Received: 26 March 2020; Accepted: 03 May 2020; Published: 08 June 2020

\begin{abstract}
In this paper, we are reporting on the comprehensive model design for time-frequency analysis system using Short-Time Fourier Transform (STFT) and Wigner-Ville Distribution (WVD) methods. As a case study, both STFT and WVD based time-frequency transforms have been developed via MATLAB platform and applied for both Chirp and Sunspot signals. The developed model considers the use of hamming moving window of length $\mathrm{L}=50$ with $90 \%$ overlapping between the current and previous window positions. The simulation results showed that WVD is more accurate method for time and frequency analysis than STFT since it can provide simultaneous localization in both time and frequency with higher resolution than STFT which can only provide localization in either time or frequency at the same time. Also, the applied techniques provide an adequate distribution of time-frequency analysis only if they used with a non-stationary signal such as Chirp signal.
\end{abstract}

Index Terms: Chirp Signal, Sunspot Signal, time-frequency Analysis, Short-Time Fourier transform (STFT), Wigner-Ville distribution (WVD), Hamming Window, non-stationary signals.

(C) 2020 Published by MECS Publisher. Selection and/or peer review under responsibility of the Research Association of Mode rn Education and Computer Science

* Corresponding author. Tel.: 615-730-0231

E-mail address: qabualha@Tnstate.edu 


\section{Introduction}

Generally, the time-frequency distribution [1] analysis of signals is to develop a joint function of time and frequency that can describe the energy density of a signal simultaneously in both time and frequency. The concept is primarily applied for non-stationary signals [2] such as Chirp signal. The non-stationary signals are those signals in which their parameters (i.e. signal parameters, spectral parameters and statistical parameters) change with time. Therefore, for any non-stationary signal which has a distinctive different frequency contents over different distinct time intervals is called a time-frequency (TF) signal and its TF distribution can be analysed by various methods such as Short-Time Fourier transform (STFT) method [3] and Wigner-Ville distribution (WVD) method [2]. However, the stationary signals in which its spectrum is time-independent such as the coloured noises and sunspot signal, they can be analysed using stationary techniques either in the time or in the frequency domain such as Discrete Fourier Transform (DFT) technique [4].

Chirp signal [5] (also known as sweep signal) sweeps linearly from a low to a high frequency (swept-frequency cosine signal) in which the frequency increases (up-chirp) or decreases (down-chirp) with time. Chirp signals are encountered in many applications ranging from radar, sonar, spread spectrum, optical communication, image processing, doppler effect, motion of a pendulum, as gravitation waves, manifestation as Frequency Modulation (FM), echo location [6]. Chirp signal is a non-stationary process signal because the event-to-event probabilities change with time (non-stochastic). Figure 1 (a) illustrates the generation of a linear chirp waveform for 500 data points. It's clearly shown that the chirp sinusoidal wave increases in frequency linearly over time. Also, Sunspot signal [7] is one of the first time-series signal that was used by Yule and Walker to develop an AR Model for sunspot cycle. For example, the sunspot time series [8] counts the average number of sunspots every year and is illustrated in Figure 1 (b) which shows yearly sunspot numbers during the period from 1700 to 2018.
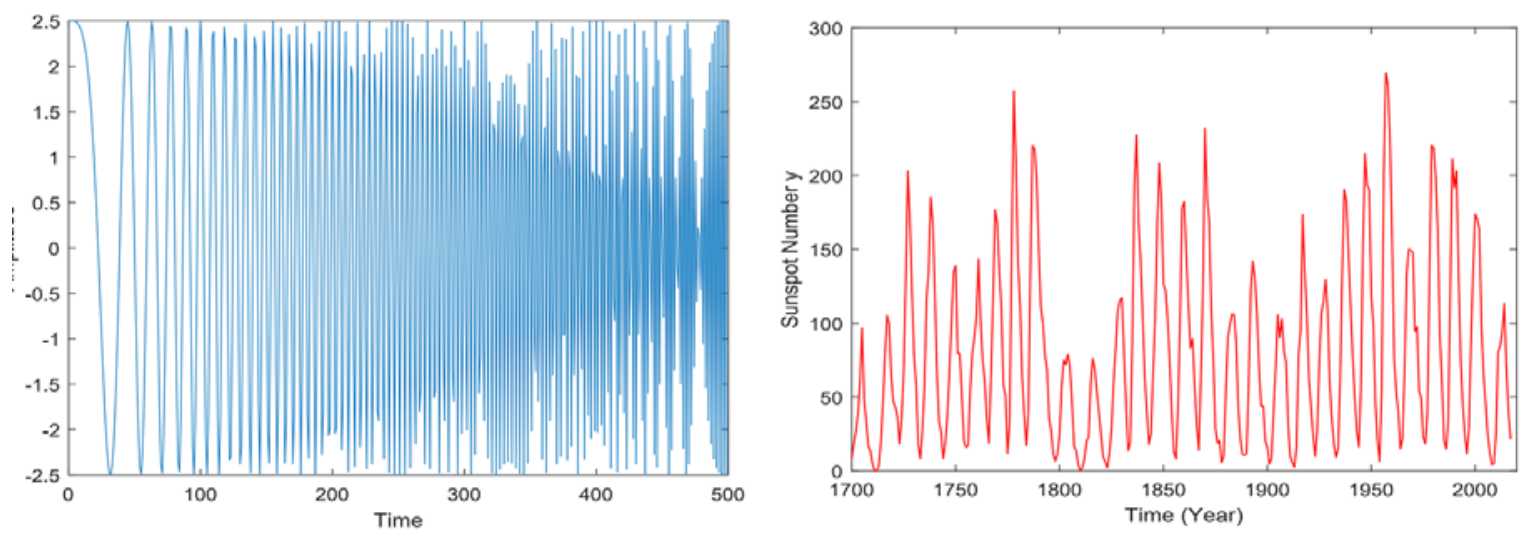

Fig. 1. (a) Chirp Signal for 500 data points (b) Time-Series for Yearly Averaged Sunspot Numbers 1700-2018

Primarily, this work provides an essential investigation and discussion for two popular Time-Frequency (TF) Analysis [9] techniques to develop a joint function of time and frequency, known as a time-frequency distribution [10], that can describe the energy density of a signal simultaneously in both time and frequency. Specifically, the main contributions of this paper can be summarized as follows:

- The familiarity with stationary signals such as Sunspot signal and non-stationary signals such as Chirp signal. 
- Developing a MATLAB codes for creating Chirp signal for 500 data points and sunspot signal for 318 data points.

- Developing a MATLAB code to implement the Short-Time Fourier transform (STFT) technique with Hamming window to analyse the time-frequency distribution for both signals (Chirp and Sunspot).

- Developing a MATLAB code to implement the Wigner-Ville distribution (WVD) technique to analyse the time-frequency distribution for both signals (Chirp and Sunspot).

- Understanding the characteristics of ideal time-frequency distribution function and the applications/signals that can be analysed with each time-frequency distribution function.

The rest of this paper is organized as follows: Section II, theoretical background, describes the overall time-frequency (TF) analysis models, features and specifications, the Short Time Fourier Transform-STFT and the Wigner-Ville Distribution-WVD. Section III, Model Development Methodology, provides the comprehensive modelling environment for the time-frequency distribution for both Chirp and Sunspot signals using both STFT and WVD. Section IV, results and Discussion presents the complete results of the TFA model implementation phase for both signals (Chirp and Sunspot) using MATLAB simulation platform (additional results are provided in the appendix). Finally, Section V, Conclusions and remarks, concludes the work in this paper.

\section{Theoretical Background}

In signal processing, time-frequency (TF) analysis [9] concerns with methods to study the signal in both the time and frequency domains simultaneously, using various TF representations (distributions) when dealing with signal whose spectral content is time-varying. In general, these methods are refinement of Fourier analysis [11] for the case when the signal frequency characteristics are varying with time such as speech, images, and medical signals. One of the most basic forms of TF analysis is the short-time Fourier transform (STFT) [12], but more sophisticated techniques have been developed, such as Wigner Ville Distribution (WVD) [13]. However, to analyse the signals well, choosing an appropriate time-frequency distribution function is important and this depends on the application being considered.

\subsection{Short Time Fourier Transform-STFT}

The Short-Time Fourier Transform (STFT) is a powerful signal processing technique that defines a time-frequency distribution for non-stationary signals whose statistic characteristics vary with time. STFT selects a number of short frames of the signal to be analysed with a window that moves with time. STFT algorithm [14] is illustrated in Figure 2 and can be summarized as follows:

\section{Algorithm: Short-Time Fourier Transform (STFT)}

1. Define analysis window (e.g., 50ms narrowband).

2. Define the amount of overlap between windows (e.g., overlapping 90\%).

3. Define a windowing function (e.g., Hamming): For hamming window, the windowing function is defined as:

$$
w[n, \tau]=0.54-0.4 \cos \left[\frac{2 \pi(n-\tau}{N_{w}-1}\right]
$$

4. Generate windowed segments: these can be generated by multiplying the signal with the window function 5. Apply the FFT to each windowed segment: Usually $\mathrm{N} \geq \mathrm{L}$, Larger $\mathrm{N}$ gives more frequency-domain samples of DTFT and thus better location and amplitude of peaks, while smaller $\mathrm{N}$ results in less computation. 


$$
Y(k)=\sum_{j=1}^{N} y(j) W_{N}^{(j-1)(k-1)} \quad \text { then } \quad y(j)=\frac{1}{N} \sum_{k=1}^{N} Y(k) W_{N}^{-(j-1)(k-1)} \quad \text { where } \quad W_{N}=e^{-2 \pi i / N}
$$

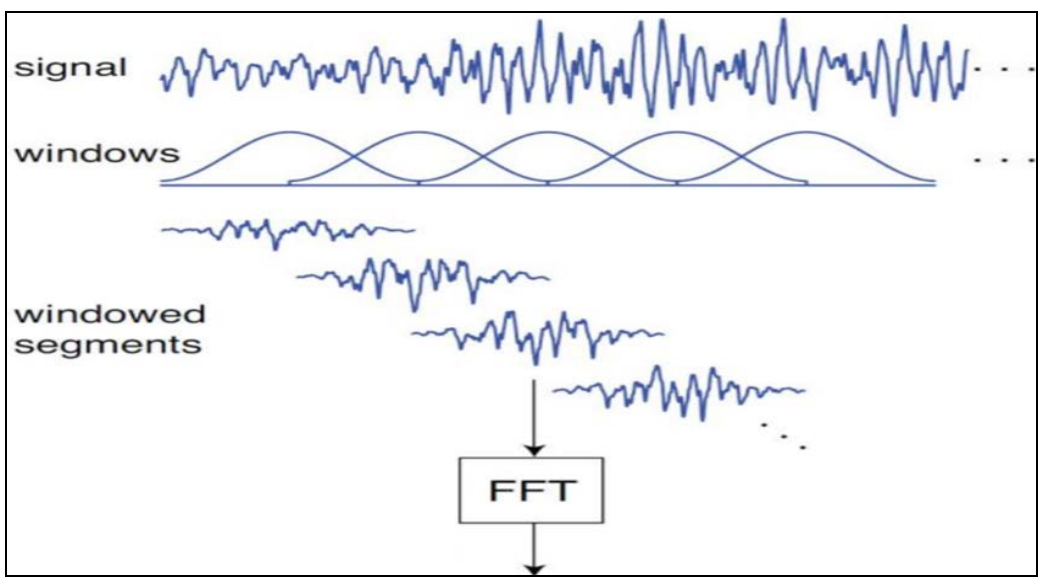

Fig. 2. Simplified view of STFT

\section{For Discrete Short-Time Fourier transform}

Here the Fourier transform of the windowed speech waveform is defined as

$$
X(n, \omega)=\sum_{m=-\infty}^{\infty} x[m] w[n-m] e^{-j \omega n}
$$

where the sequence $f_{n}[m]=x[m] w[n-m]$ is a short-time section of the speech signal $x[m]$ at time $\mathrm{n}$.

Thus, the discrete STFT can be defined as:

$$
X(n, k)=\left.X(n, \omega)\right|_{\omega=\frac{2 \pi}{N} k}
$$

Finally, the spectrogram can be defined as a graphical display of the magnitude of the discrete STFT, generally in log scale as follows:

$$
S(n, k)=\log |X(n, k)|^{2}
$$

This can be thought of as a $2 \mathrm{D}$ plot of the relative energy content in frequency at different time locations

\subsection{Wigner-Ville Distribution-WVD}

Wigner distribution (WD) is a bilinear transformation which maps a one-dimensional time-frequency signal into a two-dimensional time-frequency characterization [15]. The WD is a real valued periodic function. The rectangular windowed discrete-time WD of a signal $\mathrm{x}(\mathrm{n})$ is defined by: 


$$
W(n, \omega)=\sum_{m=-L}^{L} \omega(m) x(n+m) \bar{x}(n-m) e^{-j 2 \omega m}
$$

Where $\mathrm{n}$ is the windows time center and $\omega$ is the frequency variable. The segment window $\omega(m)$ is of width $2 L+1$ in the interval of $[n-L, n+L]$. In order to overcome the low frequency artifacts present in WD, it was Ville in 1948 who first time used the analytical signal for computing the WD and this version of WD is often referred as the Wigner-Ville Distribution (WVD) and is given by:

$$
W(n, \omega)=\sum_{m=-L}^{L} \omega(m) z(n+m) \bar{z}(n-m) e^{-j 2 \omega m}
$$

where, $z(n)$ represents analytic signal, which is complex and is calculated in the frequency domain as in the following algorithm [1]:

\section{Algorithm: Calculate analytic signal $z(n)$ for WVD}

Let $x(n)$ be the data vector of length $N$

1.Find $\mathrm{X}(\mathrm{k})$, where

$$
X(k)=F F T(x(n)) ; \quad n=1,2, \ldots . N \quad \text { and } \quad k=0,1, \ldots ., N-1
$$

2.Define $\mathrm{Z}(\mathrm{k})$, where:

$$
Z(k)= \begin{cases}X(k) & \mathrm{k}=0 \\ 2 X(k) & k=1, \ldots,(N / 2)-1 \\ 0 & \text { Otherwise }\end{cases}
$$

Thus, no aliasing is created by this method and even the sampling rate could be safely reduced to half if one exhibit any low-frequency artifact.

\section{Model Development Methodology}

In this paper, we developed a time-frequency analysis models to analyse the time-frequency distribution for Chirp signal and Sunspot activity using two common techniques, namely STFT (Short Time Fourier Transform) and WVD (Wigner-Ville Distribution). The developed models have been implemented using MATLAB computing and programming platform. However, the implementation phases of this work can be described as follows:

Phase (1): Generating the signals to be be analyzed: This phase is about generating two signals for analysis purposes, namely:

- Chirp Signal: Chirp signal can be generated linearly using the following input-output signal sinusoidal relationship formula:

$$
y(k)=A \cdot \cos [2 \pi(f(t) \cdot t)] \text { where } f(t)=f_{0}+\frac{f_{1}-f_{0}}{T} t
$$

where $A$ is the amplitude, $t$ is the current simulation time, $f_{0}$ is the initial frequency, $f_{1}$ is the target frequency, $T$ is the number of time steps between the simulation initial time and the function target time, $y(k)$ is the output signal. In this work, we generated the linear chirp signal for 500 data points $(\mathrm{k}=0,1,2,3, \ldots, 499)$ using the following signal sinusoidal formula:

$$
y(k)=2.5 \cos \left(\pi k^{2} / 1000\right)
$$

- Sunspot Signal: we have used the data set provided by the instructor which is the time series for yearly averaged sunspot numbers during the period from 1700-2018 (318 data points). 
Phase (2): Visualizing the generated signals: This phase is about plotting the original measured/generated data points for both signals before the TF modelling to see how they behave throughout the duration of time and how their values are distributed over the plane. Figure 1 shows the plots associated with this phase.

Phase (3): Developing STFT model: This phase is about applying short time frequency transform (STFT) algorithm using hamming moving window of length $\mathrm{L}=50$ with $90 \%$ overlapping between the current and previous window positions. Since STFT based on direct DFT on each windowed data, this phase was accomplished using MATLAB fft function. As a result of applying the implemented STFT algorithm on Chirp signal (Figure 1.a) and Sunspot signal (Figure 1.b), we provided two plots for Chirp signal time-frequency analysis based STFT including 3D Mesh plot in figure 3 and Spectrogram method shown in Figure 4 and one Spectrogram Plot for Sunspot signal time-frequency analysis based STFT provided in Figure 6.

Phase (4): Developing WVD model: This phase is about implementing the Wigner-Ville Distribution (WVD) algorithm for Time-Frequency Analysis discussed in the theory section of this paper. Also, this phase was accomplished using MATLAB FFT and $F F T^{-1}$ functions. As a result of applying the implemented WVD algorithm on Chirp signal (Figure 1.a) and Sunspot signal (Figure 1.b), we provided a plot for Chirp signal time-frequency analysis based WVD method shown in Figure 5 and another plot for Sunspot signal time-frequency analysis based WVD method provided in Figure 7.

Phase (5): Additional DFT implementation: This phase is about generating and plotting DFT model to show the spectral analysis of sunspot signal (Amplitude and Phase). The resulting plots of this phase are given in the Appendix of this paper.

\section{Results and Discussions}

Time-frequency (TF) analysis identifies the time at which various signal frequencies are present, usually by calculating a spectrum at regular intervals of time. In this work, we have implemented and investigated two popular algorithms, namely: STFT and WVD, to study the TF distribution for the Chirp signal (plotted in Figure 1.a) and Sunspot activity signal (Plotted in Figure 1.b). Therefore, in this section, we provide the results of the implementation phase for the developed models using MATLAB simulation platform. Figure 3 shows the linear chirp signal in time-frequency domain plotted in $3 \mathrm{D}$ mesh for better visualization and readability. Due to the linearity, non-stationary of the generated chirp signal and the proper windowing utilization, the 3D plot provided a clear joint time-frequency representation as all frequency components in this signal are clearly revealed by the used distribution.

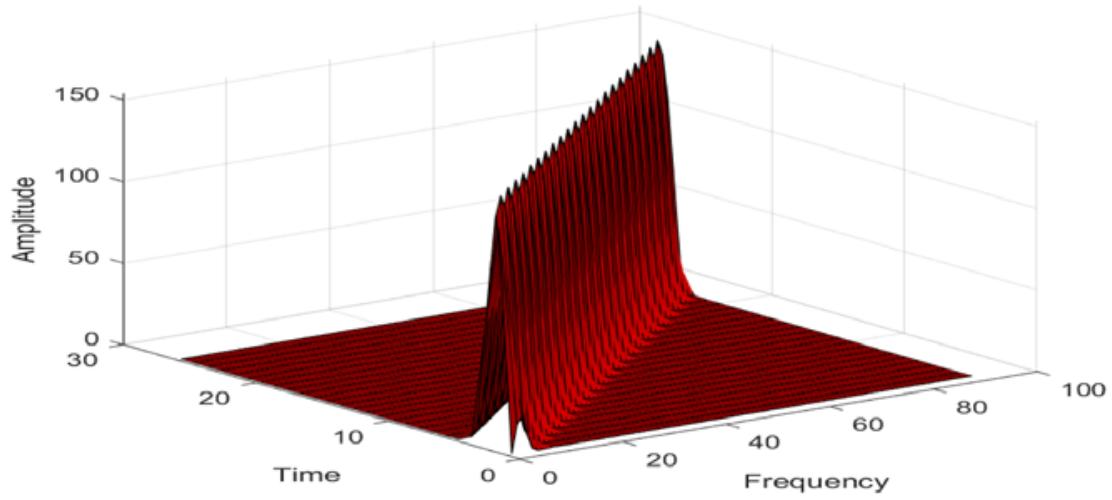

Fig. 3. Chirp signal in 3D Mesh to compare the time-frequency domain results 
Figure 4 shows the Spectrogram for Chirp signal. This plot suffers from a type of complicated aliasing that wraps in both the time and frequency axes. However, this problem has been fixed using WVD plot.

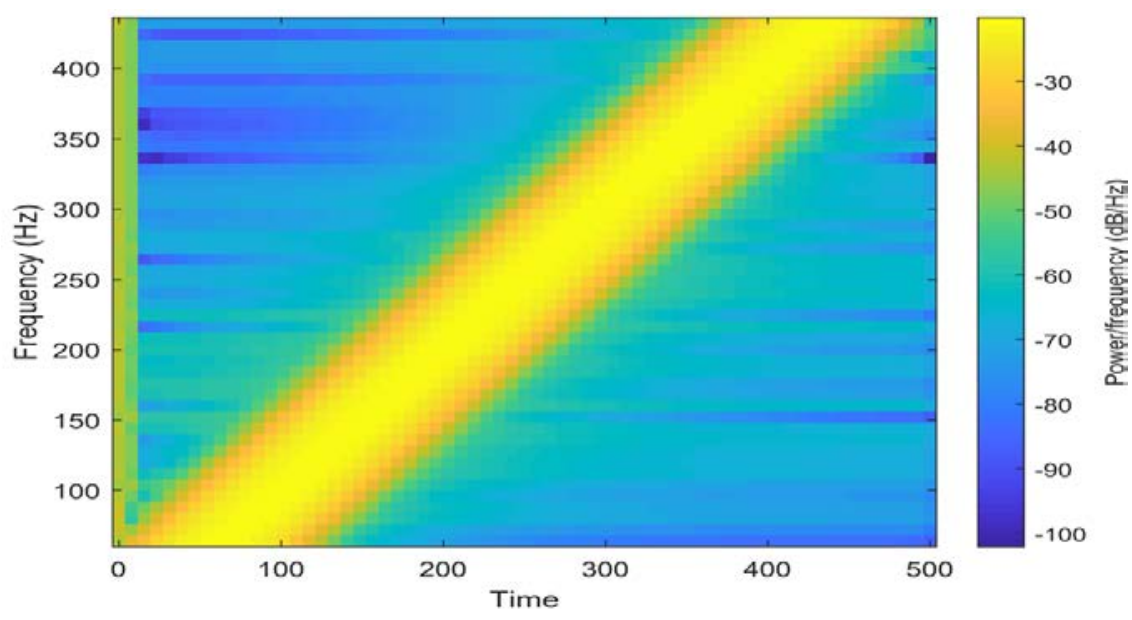

Fig. 4. spectrogram-STFT of Chirp Signal

Figure 5 shows the WV distribution for Chirp signal. This distribution fixes the problems such as the small amount of noise coming in underneath the signal and also negative energy along the line.

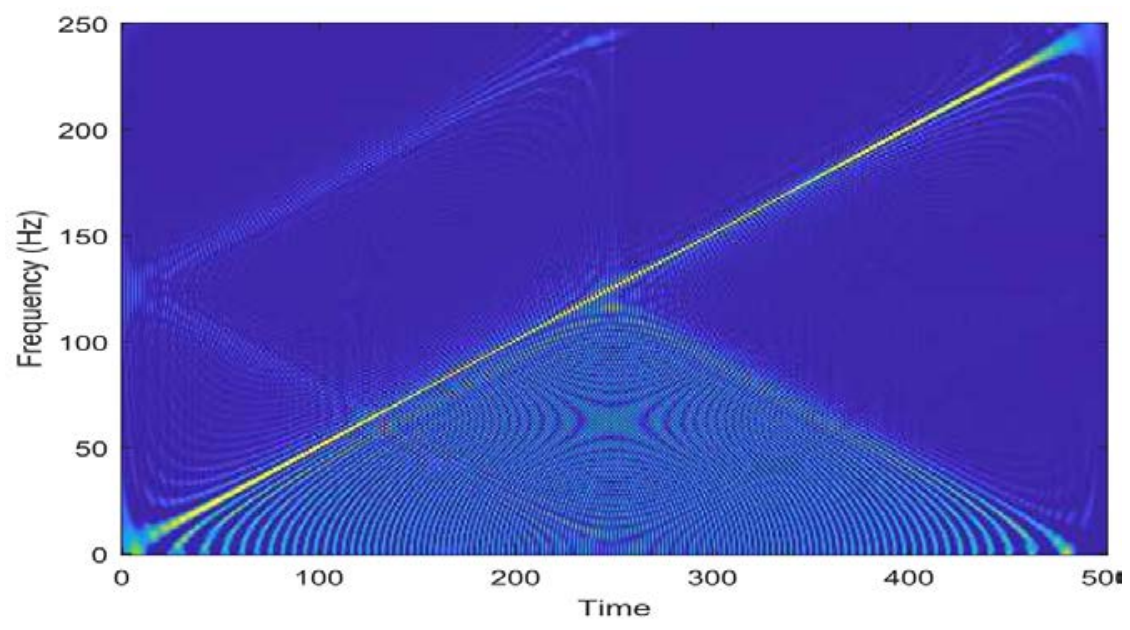

Fig.5. Wigner Ville Distribution (WVD) of Chirp Signal

Figure 6 and 7 show the spectrogram and WDV for Sunspot activity signal. Plots of both techniques illustrate an unclear behaviour of time frequency distributions. The reason of this behaviour is that the sunspot signal is a non-linear stationary signal in which its spectrum is time-independent and thus can not be analysed using non-stationary techniques. However, it can be analysed using stationary techniques either in the time or in the frequency domain such as Discrete Fourier Transform (DFT) technique. 


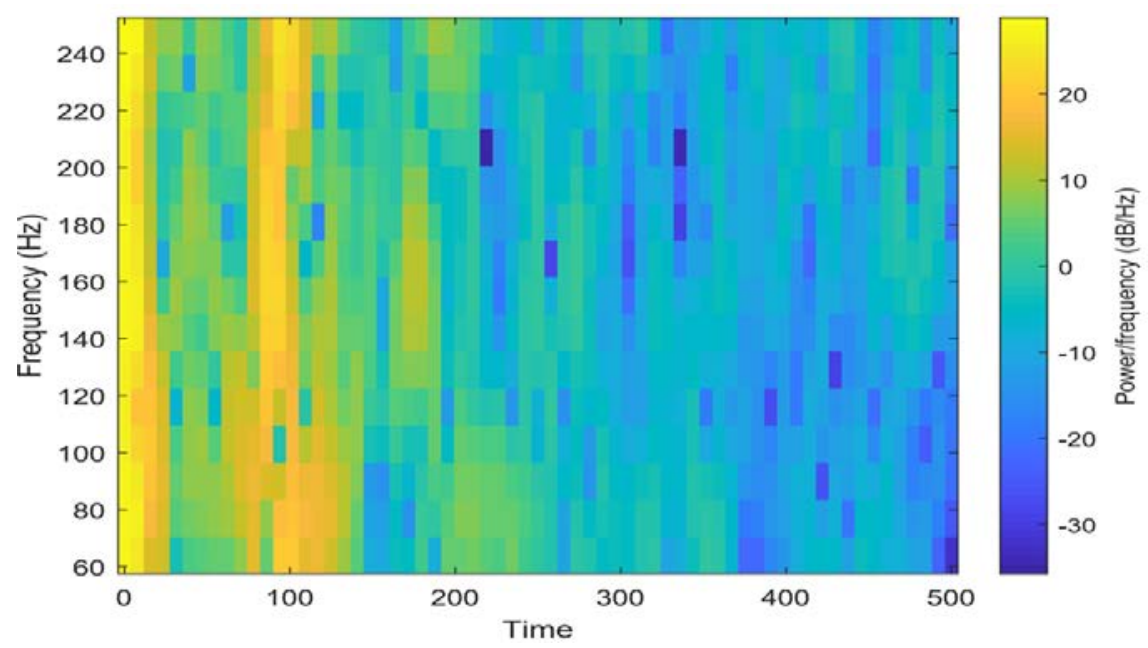

Fig. 6. spectrogram-STFT of Sunspot Signal

To sum up, the results for both techniques showed that WVD is more accurate method for time and frequency analysis than STFT since it can provide simultaneous localization in both time and frequency with higher resolution than STFT which can only provide localization in either time or frequency at the same time.

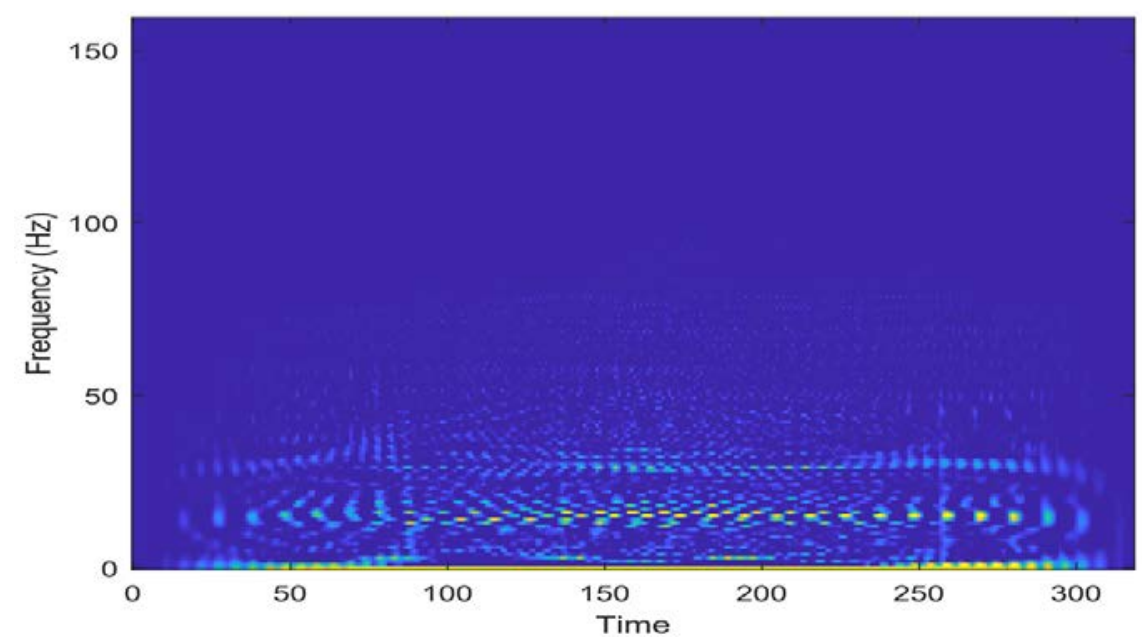

Fig. 7. Wigner Ville Distribution (WVD) of Sunspot Signal

\section{Conclusions}

Two popular time-frequency (TF) signal analysis techniques are implemented and analyzed in paper, namely, STFT and WVD. Both methods were applied to Chirp signal and Sunspot activity to develop a joint function of time and frequency (i.e. a time-frequency distribution) that can describe the energy density of a signal simultaneously in both time and frequency. The simulation results proved the advantage of using WDV technique to analyze the TF signals with no aliasing and higher resolution representation in both time and 
frequency for non-stationary signals such as Chirp signal. However, STFT is easier and faster to implement.

\section{References}

[1] Debnath L., The Wigner-Ville Distribution and Time-Frequency Signal Analysis. In Wavelet Transforms and Their Applications. Birkhauser, Boston, MA, (2002). Retrieved on-line from: https://doi.org/10.1007/978-1-4612-0097-0 5

[2] Patrick F., Time frequency and chirps. Proc. SPIE 4391, Wavelet Applications VIII, (2001). Retrieved on-line from: https://doi.org/10.1117/12.421196

[3] Sejdic E., Djurovic I. and Jiang J., Time-frequency feature representation using energy concentration: An overview of recent advances. Digital Signal Processing. 19 (1), (2009). Retrieved on-line from: https://doi.org/10.1016/j.dsp.2007.12.004

[4] J. G. Proakis and D.K. Manolakis, Digital Signal Processing. Pearson, 4th edition, ISBN-10: 0131873741, Apr 2007. Retrieved on-line from: https:/www.pearson.com/us/higher-education/program/ Proakis-Digital-Signal-Processing-4th-Edition/PGM258227.html

[5] H. Dinh, et. al. Chirp signal formats and techniques. Qualcomm Inc, 2019 US Patent \# US20190268841A1. https://patents.google.com/patent/US20190268841A1/en

[6] Li X., Bi G. Time-Frequency Filtering and Its Application in Chirp Signal Detection. In: Wu Y. (eds) Computing and Intelligent Systems. ICCIC 2011. Communications in Computer and Information Science, vol 234. Springer, Berlin, Heidelberg. https://link.springer.com/chapter/10.1007/978-3-642-24091-1_31

[7] D.J. Easterbrook. Chapter 14 - Cause of Global Climate Changes: Correlation of Global Temperature, Sunspots, Solar Irradiance, Cosmic Rays, and Radiocarbon and Berylium Production Rates, Editor(s): Don J. Easterbrook, Evidence-Based Climate Science (Second Edition), Elsevier, 2016, Pages 245-262, ISBN 9780128045886, https://doi.org/10.1016/B978-0-12-804588-6.00014-8.

[8] National Oceanic and Atmospheric Administration (NOAA), Sunspots and Solar Cycles. NOAA, Feb 2013. Retrieved on-line from: https://www.swpc.noaa.gov/phenomena/sunspotssolar-cycle

[9] Giron-Sierra J.M. Time-Frequency Analysis. In: Digital Signal Processing with Matlab Examples, Volume 1. Signals and Communication Technology. 2017. Springer, Singapore. https://doi.org/10.1007/978-981-10-2534-1_7

[10] E. Sejdić, I. Djurović, J. Jiang. Time-frequency feature representation using energy concentration: An overview of recent advances. Digital Signal Processing, vol. 19, no. 1, pp. 153-183, January 2009.

[11] P. Singh. Time-Frequency analysis via the Fourier Representation. arXiv:1604.04992, Information Theory (cs.IT); Numerical Analysis (math.NA), 2016.

[12] B. Tatsuro. Time-Frequency Analysis Using Short Time Fourier Transform. The Open Acoustics Journal. Vol.5. 2012, pages 32-38. http://10.2174/1874837601205010032.

[13] I. M. Zoukaneri and M. J. Porsani. High-resolution time frequency analysis using Wigner-Ville Distribution and the Maximum Entropy Method: Application for gas and hydrates identification. 13th International Congress of the Brazilian Geophysical Society \& EXPOGEF, Rio de Janeiro, Brazil, 2013. https://doi.org/10.1190/sbgf2013-198.

[14] R. G. Osuna, Introduction to Speech Processing. This lecture notes, CSE@TAMU, 2002. Retrieved on-line from: http://research.cs.tamu.edu/prism/lectures/sp/l6.pdf 
[15] Cao, J., Chen, G. Fast computation of Wigner Ville distribution. J. of Shanghai Univ. 7, 265-269. 2003. https://doi.org/10.1007/s11741-003-0036-5

\section{Author(s) Profile}

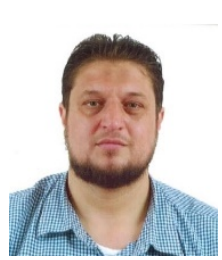

Qasem Abu Al-Haija is a PhD Candidate of Computer \& Information Systems Engineering (CISE) program at Tennessee State University, USA. Eng. Abu Al-Haija received his B.Sc. in ECE from Mu'tah University in Feb-2005 \& M.Sc. in computer engineering from Jordan university of science \& technology in Dec-2009. Research Interests: Cryptography, Cybersecurity, Cyber System Modelling \& Optimization, Computer Arithmetic, IoT and Embedded System Design.

How to cite this paper: Qasem Abu Al-Haija. “A Methodical Study for Time-Frequency Analysis Model with Experimental Case Study on Chirp Signal", International Journal of Engineering and Manufacturing (IJEM), Vol.10, No.3, pp.1-11, 2020. DOI: 10.5815/ijem.2020.03.01 


\section{Appendix A. DFT Plots for Sunspot Signal}

Discrete Fourier Transform (DFT) is one of the most important tools in Digital Signal Processing since it has a wide range of applications such as:

- DFT can calculate a signal's frequency spectrum. This is a direct examination of information encoded in the frequency, phase, and amplitude of the component sinusoids. For example, human speech and hearing use signals with this type of encoding.

- DFT can find a system's frequency response from the system's impulse response, and vice versa. This allows systems to be analysed in the frequency domain, just as convolution allows systems to be analysed in the time domain.

- DFT can be used as an intermediate step in more elaborate signal processing techniques. The classic example of this is FFT convolution, an algorithm for convolving signals that is hundreds of times faster than conventional methods.

Example: The DFT for Sunspot activity Signal is shown in Figure 8.
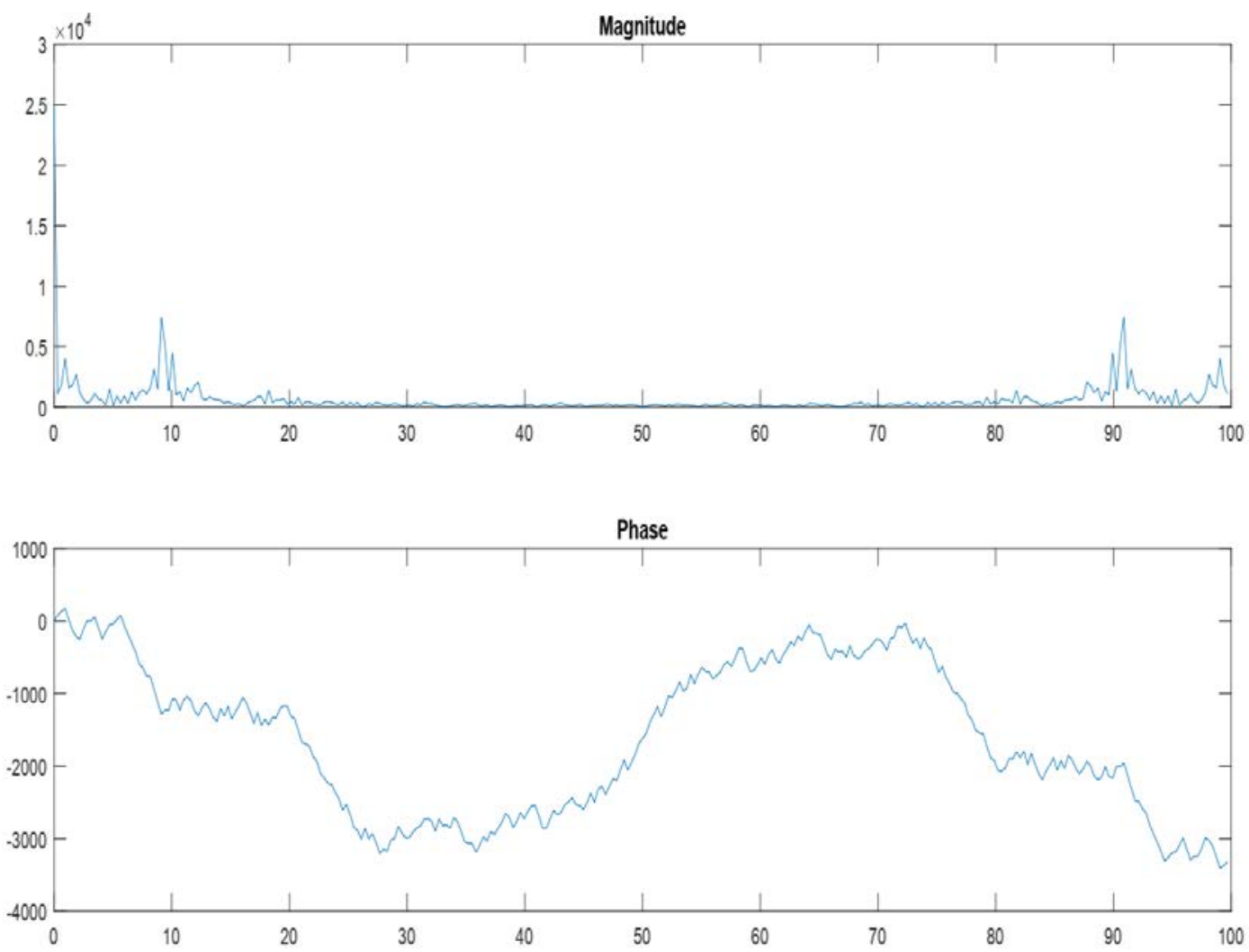

Fig. 8. DFT Analysis for Sunspot Activity Signal 